\title{
Variedades de Olivo (Olea europaea L.) de importancia económica de los departamentos Tacna y Moquegua
}

\author{
Olive varieties (Olea europaea L.) of economic importance from the departments of Tacna \\ and Moquegua \\ ${ }^{1, a, d}$ Martin Casilla-García \\ ${ }^{1, b, c}$ Mario Blas-Angulo \\ ${ }^{1, \mathrm{c}}$ Noel Catunta-Mamani \\ ${ }^{2, c . f}$ Edgar Chaparro-Aguilar
}

DIVULGACIÓN CIENTÍFICA

${ }^{1}$ Universidad Nacional Jorge Basadre Grohmann. Departamento de Agronomía. Tacna, Perú.

${ }^{2}$ Universidad Nacional Jorge Basadre Grohmann. Departamento de Ingeniería Ambiental. Tacna, Perú.

\section{Correspondencia:}

mcasillag@unjbg.edu.pe bmblasa@unjbg.edu.pe ncatuntam@unjbg.edu.pe echaparroa@unjbg.edu.pe

ORCID: 0000-0002-4882-8523 ORCID: 0000-0002-7191-3312 ORCID: 0000-0003-0750-6322

Palabras clave: Características, morfología, olivo, variedades

Keywords: Characteristics, morphology, olive, varieties

Información adicional

Presentado: 06/05/2021

Aprobado: 14/05/2021

\section{RESUMEN}

En las regiones Tacna y Moquegua existen variedades de olivo (Olea europaea L.) no caracterizadas y por ende desconocidas para la población olivicultora. Por ejemplo, la mayoría de las veces las variedades Sevillana y Empeltre son las que son cultivadas por ser las conocidas, dejando de lado otras variedades. En este trabajo se exponen quince variedades de O. europaea L. de importancia económica; las que se identificaron y describieron considerando 24 caracteres morfológicos, que corresponden a hojas (cuatro), frutos (diez) y endocarpios (diez). Se tomaron 35 muestras por variedad y se consideraron las características morfológicas en hojas (forma, tamaño, relación longitud-anchura (L/A), curvatura longitudinal), frutos (presencia y tamaño de lenticelas, diámetro, forma, simetría, forma del ápice, cavidad peduncular y lugar de inicio de envero) y en el endocarpio (forma, simetría, tamaño, posición del diámetro transversal máximo, superficie, número de surcos, distribución de surcos fibrovasculares y terminación del ápice), determinándose que las 15 variedades presentaron caracteres morfológicos diferentes.

\section{ABSTRACT}

In the Tacna and Moquegua regions, there are olive tree varieties (Olea europaea L.) that are not characterized and therefore unknown to the olive growing population, causing the Sevillana and Empeltre varieties to be cultivated, as they are known; Therefore, in the present work, fifteen varieties of $O$. europaea L. of economic importance were evaluated; those that were identified and described considering 24 morphological characters, which correspond to leaves (four), fruits (ten) and endocarps (ten). 35 samples were taken per variety and morphological characteristics were considered in leaves (shape, size, length-width ratio (L / A), longitudinal curvature), fruits (presence and size of lenticels, diameter, shape, symmetry, apex shape, pedicle cavity and place of start of veraison) and in the endocarp (shape, symmetry, size, position of the maximum transverse diameter, surface area, number of furrows, distribution of fibrovascular furrows and end of the apex), determining that the 15 varieties presented characters different morphological. 


\section{INTRODUCCIÓN}

Según las crónicas de los comentarios reales del Perú, el olivo fue introducido a las costas peruanas en 1560 desde Sevilla, España por Don Antonio de Ribera (Sotomayor, 2017). Como punto de partida de aquel entonces, comienza la expansión del olivo tanto en el norte como en el sur del Perú, en los microclimas de los valles de la costa, habiendo encontrado su óptimo desarrollo con una amplia adaptación y aceptación de los agricultores que hoy en día son los mayores productores de aceite y aceituna de mesa del país.

El olivo (O. europaea $\mathrm{L}$ ) es un árbol perennifolio que crece en promedio hasta una altura de tres a ocho metros. Presenta una copa que es redonda que puede variar de acuerdo a las podas de las ramas. También se conocen algunos de porte erguido; las ramas presentan hojas lanceoladas en su mayor porcentaje, coriáceas, dispuestas en verticilos, dímeros o pares, y las flores son de color blanco amarillento que se disponen en panículas axilares que son anuales para producir frutos (Romero et al., 2012).

La olivicultura es una actividad económica y cuenta con grandes extensiones de plantación en La Yarada-Los Palos (Pino et al., 2018). En los diferentes valles enfrenta graves restricciones en cuanto a escasez y calidad del recurso hídrico y niveles crecientes de salinidad en los suelos, por lo que desarrolla tolerancia y adaptabilidad a las condiciones medioambientales (Bastias et al., 2002). La producción de aceituna de mesa y de aceite de oliva en las irrigaciones de La Yarada - Los Palos, Magollo y Sama, ubicadas en la región Tacna, así como las de Ilo y Algarrobal de la región Moquegua, cultivadas con tecnología media y avanzada, constituyen una actividad rentable para ampliar la frontera agrícola con la introducción de nuevas variedades que se adaptan a condiciones climáticas (García \& Tello, 2006).

Según el Consejo Oleícola Internacional - COI (León, 2019), la superficie de cultivo de olivo ( $O$. europaea L.) a nivel mundial alcanza 11400000 hectáreas, instaladas en más de 50 países de los cinco continentes, el 98\% de la producción se concentra en la región Mediterránea. En el 2017, el Perú contaba con una producción de 70000 toneladas, de las que Tacna contribuyó con 69254 toneladas, contando con una superficie de 22906 ha de olivo, con rendimientos de 4800 kg/ha (Dirección Regional de Agricultura de Tacna, 2017). Existen variedades de olivo no caracterizadas y desconocidas por la población olivicultora de ambas regiones, ocasionando que se cultiven variedades como Sevillana y Empeltre, por ser más conocidas (Ramírez \& Bergamini, 2011).

Cavagnaro et al. (2001) indican que en evaluaciones de características morfológicas del olivo se deben considerar hojas, frutos, endocarpio y de ser posible el análisis a nivel molecular. En esta investigación se identificaron y describieron quince variedades de O. europaea L. de importancia económica por su rentabilidad como aceituna de mesa y materia prima para aceite de oliva (Ramírez \& Bergamini, 2011; García \& Tello, 2006), para lo que se consideraron 24 caracteres morfológicos.

El objetivo fue caracterizar e identificar quince variedades de $O$. europaea L. adaptadas a los factores abióticos característicos de zonas áridas de las regiones de Tacna y Moquegua (Chávez et al., 2001). De este modo, Como muestra de la diversidad biológica del olivo tuvo el propósito de que los residentes de sus zonas agrícolas aumentaren elsu conocimiento y conservación de las variedades de este cultivo de mucha importancia en la región e incorporen a sus buenas prácticas agrícolas (Velazco \& Velazco, 2012). la identificación basada en estas características morfológicas en sus áreas de cultivo.

\section{DESARROLLO}

El área de estudio estuvo enmarcada entre la longitud $71^{\circ} 22^{\prime}-70^{\circ} 15^{\prime}$ y latitud sur $17^{\circ} 11^{\prime}-18^{\circ} 21^{\prime}$ y a una altitud de 12 a 1174 m s. n. m. y corresponde en Moquegua a las zonas olivícolas de los distritos de Ilo, Pacocha y Algarrobal; y en Tacna, a las zonas olivícolas de los distritos de Ite, Sama Las Yaras, Sama Inclán, Tacna y los La Yarada-Los Palos.

Se visitaron 1209 parcelas olivícolas, en las que se determinó la frecuencia de las variedades cultivadas (material biológico), por referencia de sus conductores (variedades por parcela). Se evaluaron las 15 más cultivadas en la zona de estudio, que representan el 96\% del total. Se utilizaron 35 muestras de hojas, frutos y endocarpios, tomados de 35 árboles de cada variedad del material biológico, de sus cuatro 
cuadrantes de la cobertura foliar (este, oeste, norte y sur). Los materiales biológicos de estudio fueron árboles de olivo de quince variedades, en función del propósito industrial, rentabilidad económica y mayor uso referida por los agricultores del área de estudio (Tabla 1). Los materiales no biológicos utilizados en la investigación fueron el catálogo descriptor, cámara fotográfica, papel milimetrado, regla, bolsas de polietileno, tijera de podar, esteroscopio y balanza.

Para cada característica evaluada de pesos, forma de fruto y endocarpio, se realizó el análisis de correlaciones bivariadas de Pearson con el programa Jamovi Open Stats v.1.8.

\section{Tabla 01}

Variedades de olivo (O. europaea L.) de los departamentos de Tacna y Moquegua.

\begin{tabular}{|c|c|c|c|c|c|}
\hline $\mathbf{N}^{\circ}$ & Variedad & Sinonimia $^{1}$ & $\begin{array}{c}\text { Frecuencia } \\
\text { de cultivo } \\
\% \\
\end{array}$ & Propósito $^{2}$ & Origen $^{3}$ \\
\hline 1 & Arbequina & Uvilla o Arbequín & 0.9 & Aceite & España \\
\hline 2 & Ascolana & $\begin{array}{l}\text { Pecosa, Huevo de paloma y } \\
\text { Oliva dulce }\end{array}$ & 4.3 & Mesa & Italia \\
\hline 3 & Barnea & Pico de Loro & 0.6 & Aceite/Mesa & Israel \\
\hline 4 & Coratina & $\begin{array}{l}\text { Olivo confite y, en Italia, } \\
\text { La Valente y Racemo }\end{array}$ & 0.3 & Aceite & Italia \\
\hline 5 & Cornicabra & Cornudo o cuernecillo & 2.2 & Aceite & España \\
\hline 6 & Empeltre largo & $\begin{array}{l}\text { Empeltre, aragonesa, } \\
\text { Zaragozana, Terra alta }\end{array}$ & 3.1 & Aceite & España \\
\hline 7 & Farga & Común y Plateado & 2.8 & Aceite & España \\
\hline 8 & Frantoio & Machazo, tolerante & 0.6 & Aceite & Italia \\
\hline 9 & Genovesa & & 0.3 & Mesa & España \\
\hline 10 & Hojiblanca & $\begin{array}{l}\text { Casta de Lucena, } \\
\text { Lucentino, Casta de cabra }\end{array}$ & 0.3 & Aceite/Mesa & España \\
\hline 11 & Manzanilla & $\begin{array}{l}\text { Manzanilla de Sevilla, } \\
\text { Manzanilla común }\end{array}$ & 3.7 & Mesa & España \\
\hline 12 & Pendolino & Polinizador, Maurino & 0.3 & Aceite & Italia \\
\hline 13 & Picual & $\begin{array}{l}\text { Andaluza, Corriente, } \\
\text { Marteño, Martos, Picúa } \\
\text { Tacna, Criolla, Ileña, }\end{array}$ & 1.2 & Aceite & España \\
\hline 14 & Sevillana & $\begin{array}{l}\text { Gordal sevillana, Arauco, } \\
\text { Azapa }\end{array}$ & 74.0 & Mesa & Perú \\
\hline 15 & Uovo de piccione & Huevo de codorniz & 0.6 & Mesa & Italia \\
\hline
\end{tabular}

Fuente: ${ }^{1,2,3}$ Navero et al. (2000).

\section{Variables evaluadas}

En la caracterización de las variedades del Olivo, se consideraron 24 caracteres morfológicos de (cuatro) hojas, (diez) frutos y (diez) endocarpios, propuestos por Barranco (2000), Rallo (2005) y Trujillo et al. (2019), los que fueron:

Para la hoja: ( forma, tamaño, relación Largo Ancho L/A, curvatura longitudinal); para el fruto: (lugar inicio de envero, presencia o ausencia de lenticelas pequeñas o grandes, forma, simetría, tamaño, relación pulpa y endocarpio, posición del diámetro máximo, forma del ápice, base unido al pedúnculo, pezón presente o ausente, sección transversal máxima;) y para el endocarpio o hueso: (forma, simetría, tamaño, posición de perfil, diámetro del centro, base, superficie «rugosa, liso y escabrosa», número de surcos longitudinales, distribución de surcos con respecto a la base y ápice, forma de ápice redondeado o apuntado, terminación de ápice con o sin mucrón) (Ffigura 1). 

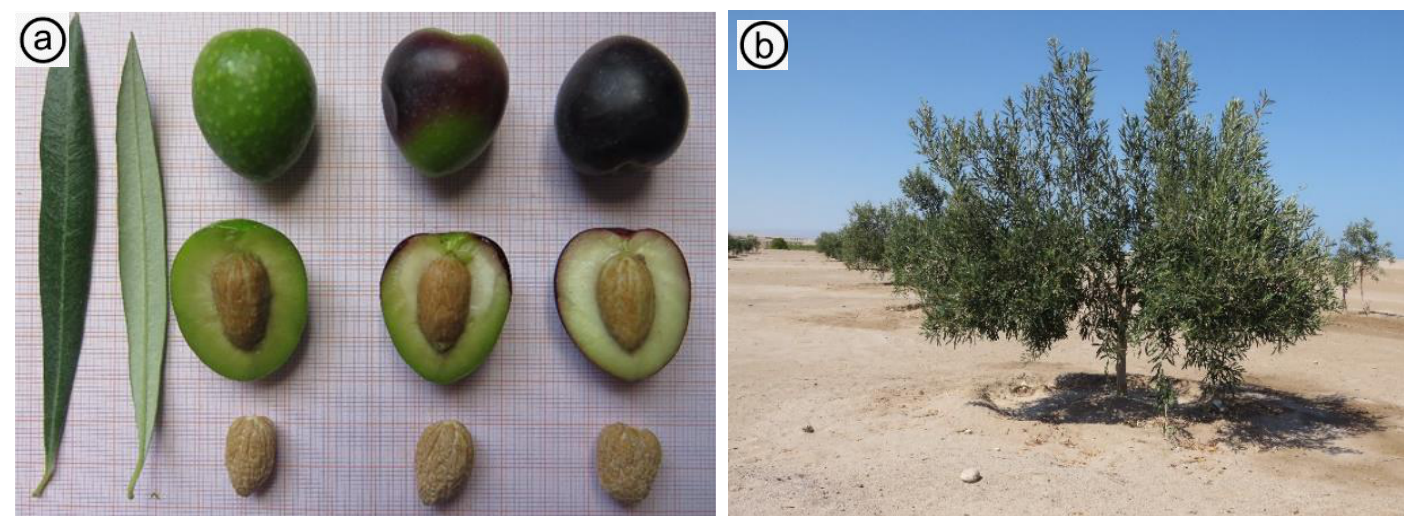

Figura 1. Caracteres morfológicos a) hojas, fruto y endocarpio; b) árbol de olivar

\section{Tabla 2}

Caracteres morfológicos de las hojas de variedades de olivo (O. europea L.)

\begin{tabular}{lllll}
\hline $\mathrm{N}^{\circ}$ & Forma del limbo & \multicolumn{1}{c}{ Longitud } & Anchura & $\begin{array}{c}\text { Curvatura longitudinal } \\
\text { del limbo }\end{array}$ \\
\hline 1 & Elíptica & Corta & Media & Epinástica \\
2 & Elíptica & Media & Media & Helicoidal \\
3 & Elíptica - Lanceolada & Media & Media & Plana \\
4 & Elíptica - Lanceolada & Larga & Media & Plana \\
5 & Elíptica - Lanceolada & Media & Media & Plana \\
6 & Elíptica - Lanceolada & Media & Media & Plana \\
7 & Elíptica & Corta & Media & Plana \\
8 & Elíptica - Lanceolada & Media & Media & Plana \\
9 & Lanceolada & Media & Media & Plana \\
10 & Lanceolada & Larga & Media & Plana \\
11 & Elíptica & Media & Media & Plana \\
12 & Lanceolada & Media & Media & Epinástica \\
13 & Elíptica & Media & Media & Plana \\
14 & Elíptica - Lanceolada & Larga & Media & Plana \\
15 & Elíptica - Lanceolada & Larga & Ancha & Plana
\end{tabular}

Nota: Forma (cm): Elíptica (L/A < 4), Elíptico- lanceolada (L/A 4- 6) y Lanceolada (L/A > 6); Longitud: Corta ( $<5)$, Media (5- 7) y Larga ( $>7)$; Ancho: Estrecha $(<1)$, Media $(1-1,5)$ y Ancha $(>1,5)$. 


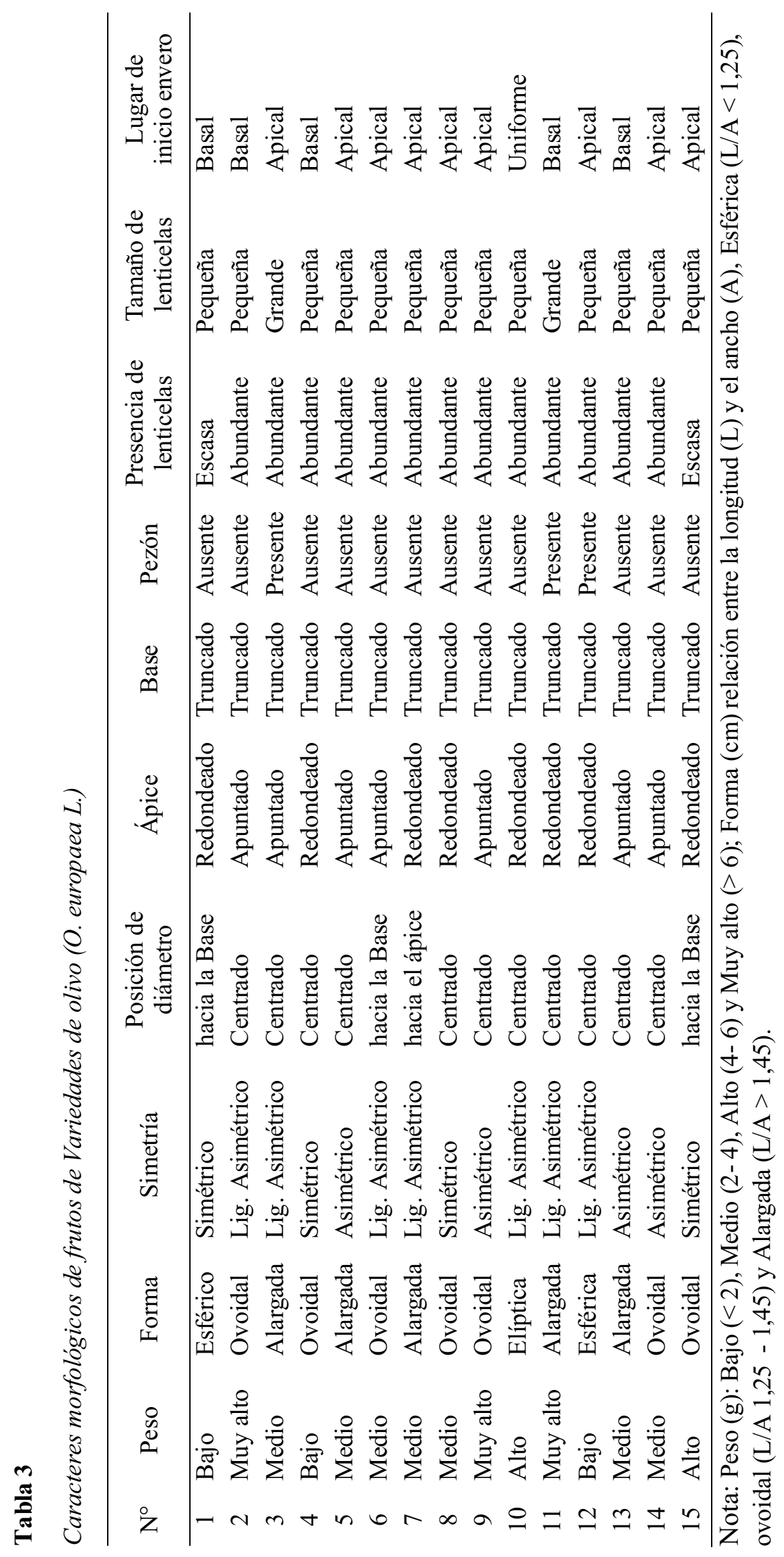

Ciencia \& Desarrollo, Vol. 20, Núm. 1, pp. 87-95 (2021) 


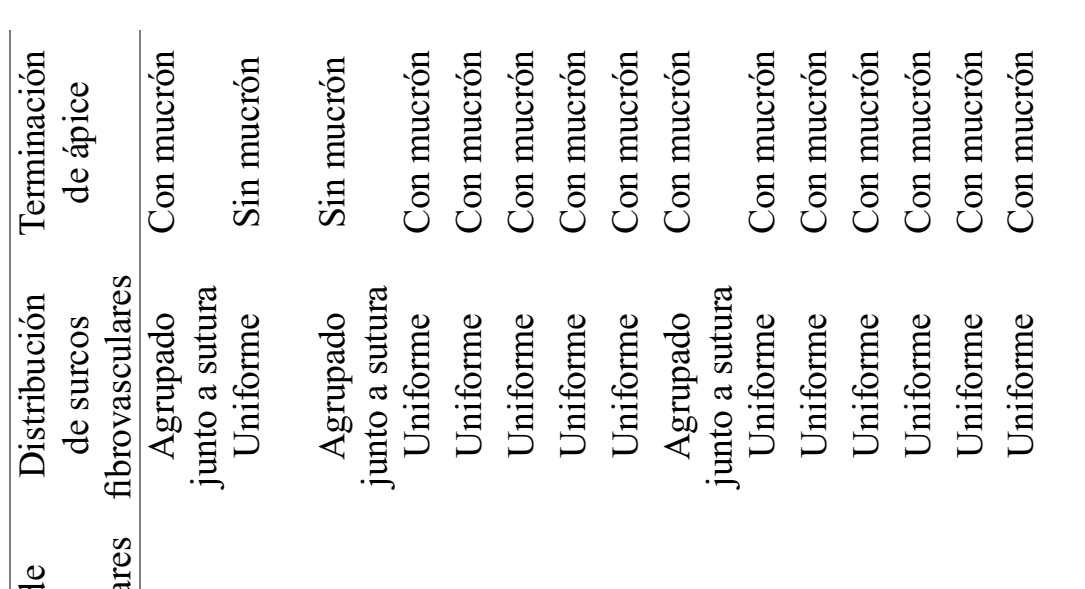

总

空

ᄀ

을

त 1

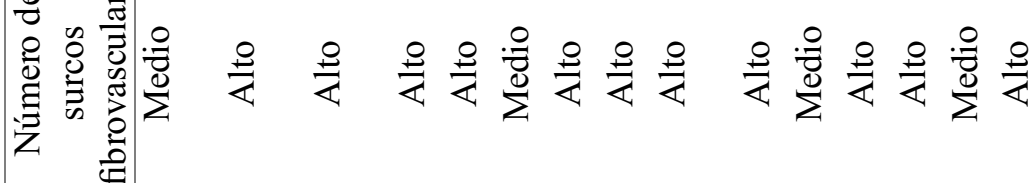

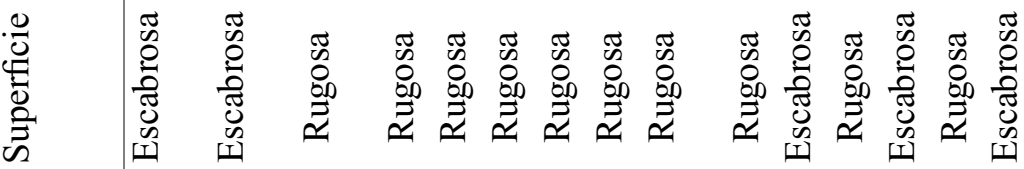

$>.9$

已

蛋

कृ $v$

$\frac{\pi}{\pi} \cdot \frac{0}{\oplus}$

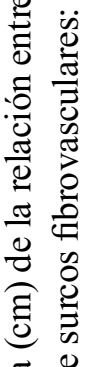

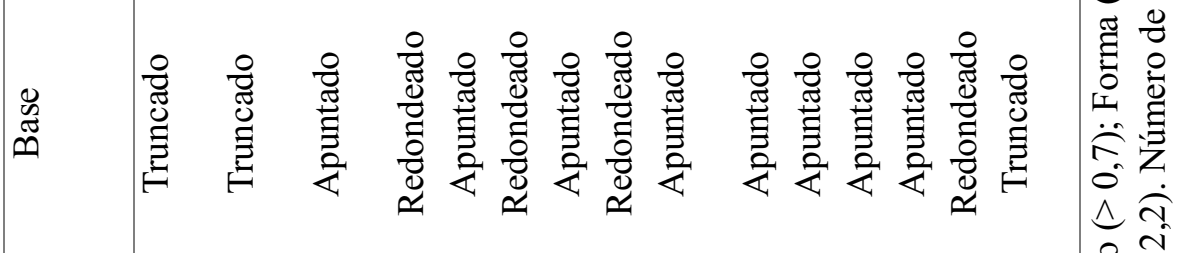

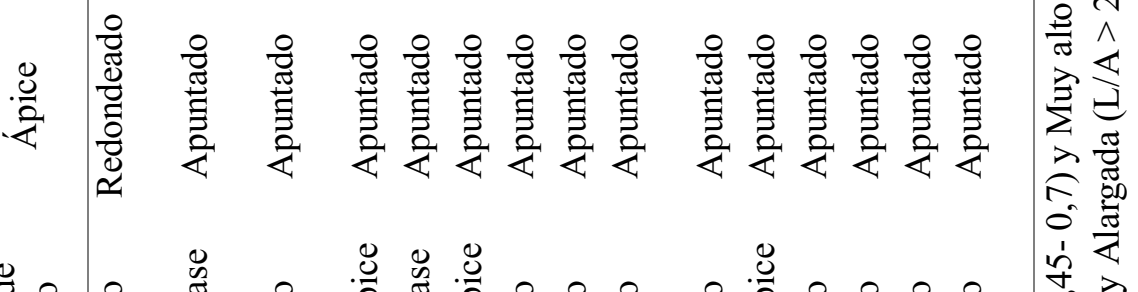

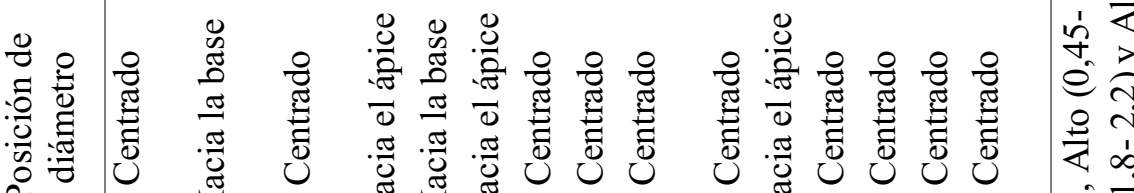

ᄋ

$\stackrel{8}{?}$

\&

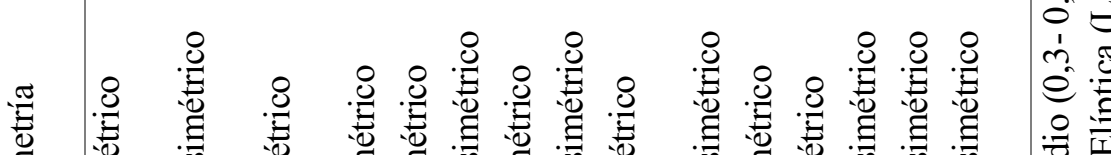

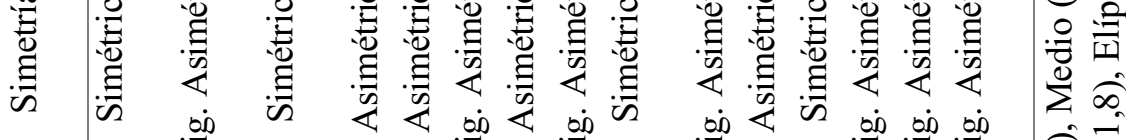

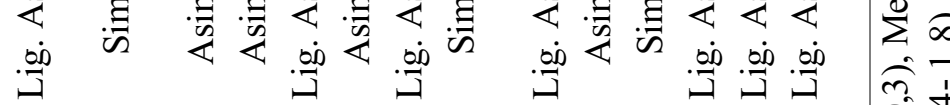

8

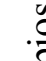

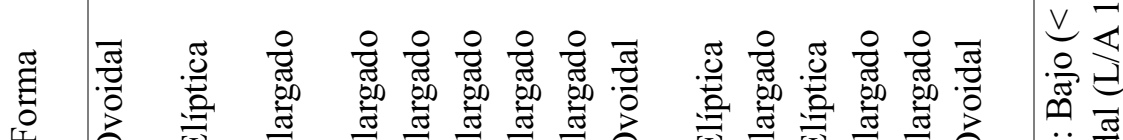

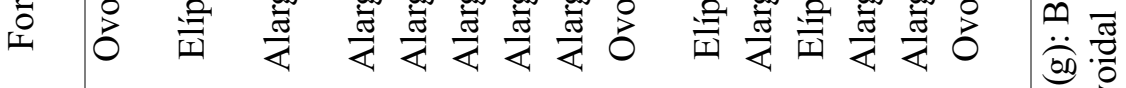

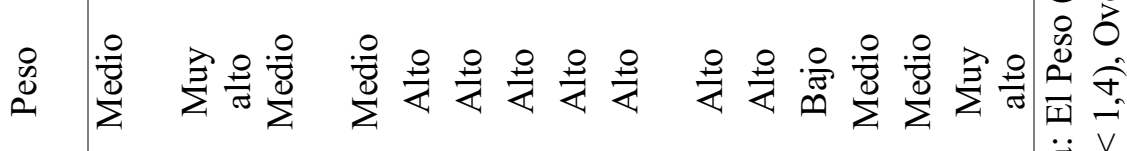

齐

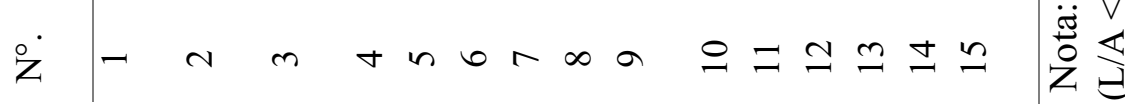

92 Ciencia \& Desarrollo, Vol. 20, Núm. 1, pp. $87-95$ (2021) 
Tabla 5

Correlación de peso, forma, tamaño y endocarpio de frutos de olivo (O. europaea L.)

\begin{tabular}{|c|c|c|c|c|c|c|c|}
\hline \multirow[t]{2}{*}{$\mathrm{N}^{\circ}$} & \multirow{2}{*}{ Variedades } & \multicolumn{6}{|c|}{ Caracteres Correlacionales } \\
\hline & & $\mathrm{Pp} / \mathrm{Pe}$ & $\mathrm{Pp} / \mathrm{Fe}$ & $\mathrm{Pe} / \mathrm{Fe}$ & $\mathrm{Pf} / \mathrm{Ff}$ & $\mathrm{Pf} / \mathrm{Fe}$ & $\mathrm{Ff} / \mathrm{Fe}$ \\
\hline 1 & Arbequina & $0.550^{* *}$ & $0.265^{\mathrm{ns}}$ & $0.372 *$ & $0.119^{\mathrm{ns}}$ & $0.284^{\mathrm{ns}}$ & $0.241^{\mathrm{ns}}$ \\
\hline 2 & Ascolana & $0.814^{* *}$ & $-0.374 *$ & $-0.260^{\text {ns }}$ & $0.200^{\mathrm{ns}}$ & $-0.370 *$ & $-0.280^{\text {ns }}$ \\
\hline 3 & Barnea & $0.349^{*}$ & $-0.323^{\text {ns }}$ & $-0.419 *$ & $-0.454 *$ & $-0.341^{\mathrm{ns}}$ & $-0.076^{\mathrm{ns}}$ \\
\hline 4 & Coratina & $0.944 * *$ & $-0.656^{* *}$ & $-0.416^{* *}$ & $-0.027^{\mathrm{ns}}$ & $-0.619 * *$ & $-0.338^{*}$ \\
\hline 5 & Cornicabra & $0.726^{* *}$ & $0.341 *$ & $0.521 * *$ & $-0.244^{\mathrm{ns}}$ & $0.369 *$ & $-0.481 * *$ \\
\hline 6 & Empeltre & $0.759^{* *}$ & $0.886^{* *}$ & $0.664^{* *}$ & $-0.024^{\mathrm{ns}}$ & $0.881^{* *}$ & $0.218^{\mathrm{ns}}$ \\
\hline 7 & Farga & $0.666^{* *}$ & $0.544^{* *}$ & $0.195^{\mathrm{ns}}$ & $0.274^{\mathrm{ns}}$ & $0.531 * *$ & $0.480^{* *}$ \\
\hline 8 & Frantoio & $0.160^{\mathrm{ns}}$ & $0.125^{\text {ns }}$ & $0.742 * *$ & $-0.261^{\mathrm{ns}}$ & $0.192^{\text {ns }}$ & $0.117^{\mathrm{ns}}$ \\
\hline 9 & Genovesa & $0.705^{* *}$ & $0.495^{* *}$ & $0.29^{\mathrm{ns}}$ & $-0.062^{\mathrm{ns}}$ & $0.486^{* *}$ & $0.226^{\mathrm{ns}}$ \\
\hline 10 & Hojiblanca & $-0.263^{\text {ns }}$ & $0.744 * *$ & $0.018^{\mathrm{ns}}$ & $0.176^{\text {ns }}$ & $0.762 * *$ & $0.452 * *$ \\
\hline 11 & Manzanilla & $0.591^{* *}$ & $0.053^{\text {ns }}$ & $0.278^{\mathrm{ns}}$ & $0.150^{\mathrm{ns}}$ & $0.072^{\mathrm{ns}}$ & $0.156^{\mathrm{ns}}$ \\
\hline 12 & Pendolino & $0.529^{* *}$ & $-0.278^{\mathrm{ns}}$ & $-0.466^{* *}$ & $-0.368^{*}$ & $-0.344^{*}$ & $0.280^{\mathrm{ns}}$ \\
\hline 13 & Picual & $0.591 * *$ & $0.265^{\mathrm{ns}}$ & $-0.259^{\mathrm{ns}}$ & $-0.158^{\text {ns }}$ & $0.204^{\text {ns }}$ & $-0.103^{\text {ns }}$ \\
\hline 14 & Sevillana & $-0.536^{* *}$ & $-0.162^{\mathrm{ns}}$ & $0.483^{* *}$ & $-0.006^{\mathrm{ns}}$ & $-0.111^{\mathrm{ns}}$ & $0.536^{* *}$ \\
\hline 15 & $\begin{array}{l}\text { Uovo de } \\
\text { piccione }\end{array}$ & $0.421 *$ & $0.605^{* *}$ & $0.529 * *$ & $0.57 * *$ & $0.63 * *$ & $0.55 * *$ \\
\hline
\end{tabular}

Nota: Pp/Pe: Peso de pulpa/Peso de endocarpio; Pp/Fe: Peso de pulpa/Forma de endocarpio; Pe/Fe: Peso de endocarpio/ Forma de endocarpio; Pf/Ff: Peso de fruto/Forma de fruto; Pf/Fe: Peso de fruto/Forma de endocarpio; Ff/Fe: Forma de fruto/Forma de endocarpio; ns: no significativo

La forma de limbo dominante es la elíptica-lanceolada. La longitud que resalta en las variedades de olivo es la media, por lo que corresponde a un carácter diferenciador de Arbequina, Coratina, Farga y Frantoio. La característica 'ancho de la hoja', ns sirve particularmente para determinar la variedad Uovo de piccione que tiene un valor mayor que $1 ., 5 \mathrm{~cm}$ (ancha), en tanto las demás presentan un valor entre $1-1 ., 5 \mathrm{~cm}$ (media) (Tabla 2). El carácter curvatura longitudinal del limbo, la plana es la dominante, por lo que este carácter nos ayuda a diferenciar las variedades Arbequina, Ascolana y Pendolino. En general, las características longitud y anchura de las hojas definen el tamaño que en general es grande, resultados similares a los que obtuvieron García \& Tello (2006), así como los de Molano \& González (2014) que determinaron que las hojas miden generalmente entre 4.,6 y 7.,0 cm de largo y entre 1.,0 y $1 ., 5 \mathrm{~cm}$ de ancho con forma lanceolada.

Según Rallo (1994), el olivo como otras plantas frutales presentan una relación negativa entre la cantidad de frutos por árbol y el peso que presenta el fruto, por lo que para esta especie los valores que superan los seis gramos se consideran muy altos, y sirven como diferenciador de las variedades Ascolana, Genovesa y Manzanilla; en tanto que Coratina y Pendolino presentan valores menores que dos gramos. Todos los frutos poseen una base truncada por lo que este carácter no se utiliza como criterio diferenciador entre estas quince variedades. Barnea, Manzanilla y Pendolino presentan pezón. Las variedades Arbequina y Uovo de Piccione muestran escases de lenticelas.

El endocarpio es la parte interna de los frutos que muestra características únicas según la variedad de olivo (Barranco et al., 2007), se forma en el proceso de esclerificación presentando características diferenciadoras conforme madura el fruto (Rallo, 1994)., Een el trabajo resaltan los indicadores del ápice redondeado de Arbequina a diferencia del resto de variedades que presentan ápice apuntado.; Aasimismo, la terminación del ápice sin mucrón en Ascolana y Barnea, y con mucrón en las demás variedades de olivo (Tabla 4), coincidentes con lo definido por Barranco et al. (2000). 
De las 15 variedades de importancia económica encontradas y analizadas de los departamentos de Tacna y Moquegua, todas presentan aspectos morfológicos con una combinación particular de caracteres que los diferencian de las demás; así, las reportadas por Romero et al. (2012) como variedades de Catamarca, Argentina (Arbequina, Arauco, Manzanilla, Picual, Siria, Barnea, Piccioline Marroquí, Carolea y Coratina) son distintas a las que se ubican en Tacna y Moquegua.

Las características morfológicas que se evaluaron en hojas frutos y endocarpio de las diferentes variedades de olivo durante su desarrollo desde la formación de frutos hasta su maduración tienen un período entre a 6 a 8 meses (Barranco et al., 2000). La forma de la hoja es un carácter discriminante (elíptica, elíptica-lanceolada y lanceolada); asiasí comomismo la simetría del fruto, forma del ápice y base del fruto, posición del diámetro y número de surcos fibrovasculares que se observan. (Tabla 2, 3, y 4), siendo importante considerar para la identificación la hoja, el fruto y el endocarpio.

Las variedades evaluadas fueron Ascolana, Barnea, Coratina, Cornicabra, Empeltre, Farga, Frantoio, Genovesa, Hojiblanca, Manzanilla, Pendolino, Picual, Sevillana y Uovo de piccione. Se hace una comparación con las variedades evaluadas por García y Tello (2006) que fueron Sevillana (clon), sevillana (injertada), Limoncillo, Cornezuelo, Koservolia, Uovo di piccione, Manzanilla de Sevilla, Campanil, Azapa, Azedj, Picholine, Arauco y Leccino que están ubicadas en el banco de germoplasma del Instituto Basadre de Investigación en Agrobiotecnología y Recursos Genéticos- IRGAB, de la Universidad Nacional Jorge Basadre Grohmann, que sirven para estudios y preservación de variedades, siendo algunas de importancia económica o biológica.

En los factores estudiados existe aleatoriedad correlacional, esto permite inferir que existe una manifestación heterogénea en cada variedad evaluada. En algunos de los caracteres confrontados de cada variedad se evidencia una correlación ya sea positiva o negativa sin seguir un patrón o una secuencia lógica. Entonces, en base al análisis bivariado de Pearson y la correlación resultante, los factores a considerar son, en primer lugar, el Peso de pulpa/peso de endocarpio, seguido de Peso de pulpa/Forma de endocarpio y Peso de fruto/Forma de endocarpio semejante a lo detallado por Barranco et al. (2000). La correlación lineal positiva y negativa se sugiere a la particularidad que presenta cada variedad con características únicas que la diferencian de otras en esta la evaluación (Tabla 5).

Las caracterizaciones mencionadas de las variedades permitieron analizar las bivariantes morfológicas de frutos de 20 caracteres morfológicos. Los caracteres fueron analizados y validados en las condiciones edafoclimáticas de los departamentos de Tacna y Moquegua reportadas por Sakoda \& Tonietto (2013).

\section{CONCLUSIONES}

Dentro de las características de mayor importancia en la diferenciación de variedades se encuentran aquellas que predominan como la forma elíptica-lanceolada del limbo, la longitud media de la hoja, las hojas anchas, curvatura longitudinal plana, el peso alto y el pezón de los frutos; la presencia del mucrón y el ápice apuntado del endocarpio.

Los factores relacionales más importantes a considerar en la determinación varietal son el Peso de pulpa/peso de endocarpio, Peso de pulpa/Forma de endocarpio y Peso de fruto/Forma de endocarpio.

Las 15 variedades presentes en el área de estudio e identificadas mediante las características morfológicas de sus frutos, endocarpios y hojas son la Arbequina, Ascolana, Barnea, Coratina, Cornicabra, Empeltre, Farga, Frantoio, Genovesa, Hojiblanca, Manzanilla, Pendolino, Picual, Sevillana y Uovo de Piccione.

\section{AGRADECIMIENTOS}

Agradecemos a la Universidad Nacional Jorge Basadre Grohmann (Tacna, Perú) por la asignación de los fondos de canon y sobrecanon minero al proyecto «Inventario y prospección del olivo (Olea europaea 
L.) a través de descriptores morfológicos en los departamentos de Moquegua y Tacna», con Resolución Rectoral No 5975-2019-UN/JBG.

\section{REFERENCIAS}

Barranco, D., Fernández Escobar, R., \& Rallo Romero, L. (2007). El cultivo del olivo $6^{a}$ ed. Mundi-Prensa Libros.

Barranco, D., Cimato, A., Fiorino, P., Rallo, L., Touzani, A., Castañeda, C., Serafini, F., \& Trujillo, I. (2000). Catálogo mundial de variedades de olivo. Consejo Oleícola Internacional, Madrid, Spain, 360.

Bastías, M., Escobar, A., Carrasco, R., Figueroa, T., \& Tapia, I. (2002) Caracterización de las relaciones hídricas y respuestas fisiológicas en plantas de olivo (Olea europaea L.) cultivadas en condiciones de salinidad, 1 Región. Idesia, 20(2).

Cavagnaro, P., Juárez, J., Bauza, M., \& Masuelli, R. W. (2001). Discriminación de variedades de olivo a través del uso de caracteres morfológicos y de marcadores moleculares. Agriscientia, 18.

Chavez, R., Casilla, E., Salazar, L., \& Bartolini, I. (2001). Hacia la identificacion y control integrado de hoja de hoz en los cultivos de olivo de Tacna y Arica. Serie Actas-Instituto de Investigaciones Agropecuarias.

García, R. D. A., \& Tello, M. S. R. (2006). Caracterización morfológica de trece variedades de olivo (Olea europaea L.) introducidas en el germoplasma del INPREX-Tacna. Ciencia \& Desarrollo, 10, $107-110$.

León, J. C. (2019). Radiografía mundial del olivo. Agencia agraria de noticias.

Molano, J. F. G., \& González, E. Y. C. (2014). Comportamiento fenológico del olivo (Olea europaea L.) en el alto ricaurte (Boyacá). Revista Logos, Ciencia \& Tecnología, 6(1), 1-13.

Navero, D. B., Cimato, A., Fiorino, A., Fiorino, P., Romero, L. R., Touzani, A., Castañeda, C., Serafini, F., \& Navas, I. T. (2000). Catalogo mundial de variedades de olivo. Madrid: Consejo Oleicola Internacional.

Pino, E., Chávarri, E., \& Ramos, L. (2018). Crisis de gobernanza y gobernabilidad y sus implicancias en el uso inadecuado del agua subterránea, caso acuífero costero de La Yarada, Tacna, Perú. Idesia (Arica), 36(3), 77-85.

Rallo, L. (1994). Fructificación y producción en olivo. Agricultura, 746, 13-16.

Rallo, L. (2005). Variedades de olivo en España: una aproximación cronológica. In: Rallo, L., Barranco, D., Caballero, J., del Río, C., Martin, A., Tous, J., Trujillo, I. eds. Variedades de olivo en España. Madrid, Mundi-Prensa, 17-44.

Ramírez, E. A. D. F., \& Bergamini, L. del C. L. (2011). Determinación de la Aptitud para la Obtención de Aceite de Oliva de Seis Variedades de Aceituna Negra (Olea europaea L.) del Banco de Germoplasma del INPREX UNJBG Tacna. Ciencia \& Desarrollo, 13, 50-53.

Romero, N., Saadi, L., Jorratti, M., \& Andrada C. (2012). Estudios morfológicos de variedades de olivo (Olea europaea L.) del valle central de la provincia de Catamarca. Revista Biología en Agronomía, 2 (1).

Sakoda, B. H., \& Tonietto, J. (2013). El clima vitícola de regiones productoras de uvas para vinos y piscos del Perú. Clima, zonificación y tipicidad del vino en regiones vitivinícolas iberoamericanas, 299.

Sotomayor León, E. M. (2017). El olivo (Olea europaea L) en América. Idesia (Arica), 35(3), 3-6.

Trujillo, I., Barranco, D., Cabello, D., Gordon, A., Morello, P., Muñoz Diez, C., \& Rallo, L. (2019). Proposal of a guide for the catalogation, sanitation and management of the ioc network (UCOLIVO, 1. Ucolivo.

Velazco, J., \& Velazco, J. (2012). Características del empleo agrícola en el Perú. Empleo y protección social, $1,161-211$. 